\title{
Transient optical angular momentum effects and atom trapping in multiple twisted beams
}

\author{
D. L. Andrews*, A. C. Carter ${ }^{* *}$, M. Babiker ${ }^{* *}$ and M. Al-Amri*** \\ ${ }^{*}$ School of Chemical Sciences, University of East Anglia, Norwich NR4 7TJ, U.K \\ ** Department of Physics, The University of York, York YO10 5DD, U.K. \\ *** Department of Physics, King Khalid University, Abha, P.O.B. 9003, Saudi Arabia
}

\begin{abstract}
Theoretical work has already established the existence of a light-induced torque acting on the centre of mass of an atom, ion or molecule immersed in twisted light, where the transition frequency is suitably detuned from that of the twisted light beam. The twisted beam carries $\ell$ units of orbital angular momentum per photon, and the steady-state saturation form of the torque is also determined by the width of the upper state in the atomic transition. It has been shown that, to leading order, the transfer of orbital angular momentum can only occur between the twisted light and the centre of mass motion. We argue here that, for small linewidth, the full time-dependence of the torque is needed to account correctly for the dynamics of atoms in a twisted light beam. We outline the theoretical framework needed to derive this full timedependence, applying the theory to the motion in a twisted light beam of $\mathrm{Eu}^{3+}$ ions, which possess a particularly narrow linewidth state. For relatively large linewidth, the steady-state forces and torque are appropriate, but the processes of cooling and trapping require the application of several suitably oriented twisted beams. The description of atomic motion in multiple twisted beams demands the application of special coordinate transformations. We show how to construct the appropriate transformation matrices to represent a twisted light beam propagating in an arbitrary direction, and we proceed to investigate the cooling and trapping of $\mathrm{Mg}^{+}$ions in sets of pairs of counter-propagating twisted beams in two-dimensional and three-dimensional molasses configurations.
\end{abstract}

Keywords: Optical vortices, twisted beams, optomechanical effects, optical cooling, optical trapping, optical molasses

\section{INTRODUCTION}

In a wide range of laser applications for optical manipulation, the translational motion of small particles is controlled through radiation forces exerted by the laser light. Such principles can be extended to nanoscale objects such as atoms. ${ }^{1-3}$ When a laser beam has a structure that is endowed with orbital angular momentum, as for example with the well-studied Laguerre-Gaussian 'twisted' modes, there is in addition an optically induced torque that leads to a rotational motion of the atoms about the beam axis. ${ }^{4-10}$ This torque has been the subject of several investigations over the last decade or so. ${ }^{11,12}$ The trajectory of a given atom in a Laguerre-Gaussian light beam obviously depends on the initial conditions at the instant the beam is switched on. Most treatments of such radiation effects on atoms assume that steady-state position-dependent forces and associated torques are operative at the outset and during the entire motion; however, this assumption is not justifiable in general. We have found that the full time-dependence is characterized by a transient regime in which the atoms are subject to forces and torques that differ markedly from their steady-state forms, giving rise to new features in the subsequent dynamics, in both the translational and rotational aspects of the motion. ${ }^{13}$ To demonstrate the importance of the transient regime we apply the theory to the case of narrow-linewidth $\mathrm{Eu}^{3+}$ ions in a Laguerre-Gaussian beam. In the case of atoms with a relatively large linewidth, a consideration of steadystate forces and torques is arguably more appropriate, but processes of cooling and trapping can require the application of several, suitably oriented, twisted beams. This introduces into the theory an additional level of mathematical complexity, as it is necessary to construct appropriate orientational transformation matrices to represent a twisted beam propagating in an arbitrary direction. Here we demonstrate the method, also illustrating the ensuing results through application to the cooling and trapping of $\mathrm{Mg}^{+}$ions in pairs of counter-propagating twisted beams, in two-dimensional as well as three-dimensional configurations. 


\section{TRANSIENT DYNAMICS IN A SINGLE TWISTED BEAM}

\section{A. Beam characteristics}

We shall be concerned with atomic motions in the electromagnetic field of a general Laguerre-Gaussian (LG) laser beam. For such a beam, the electric field operator can be expressed in quantized form as follows:

$$
\mathbf{E}(\mathbf{R})=\mathrm{i}\left[a \hat{\varepsilon} F_{k l p}(\mathbf{R}) e^{\mathrm{i} \Theta_{k l p}(\mathbf{R})}-\text { h.c. }\right] \text {, }
$$

where $\hat{\varepsilon}$ is a mode polarization vector, while $F_{k l p}(\mathbf{R})$ and $\Theta_{k l p}(\mathbf{R})$ are the mode amplitude and phase, respectively;

$$
\begin{gathered}
F_{k l p}(\mathbf{R})=F_{k 00} \frac{N_{l p}}{\left(1+z^{2} / z_{R}^{2}\right)^{1 / 2}}\left(\frac{\sqrt{2} r}{w(z)}\right)^{|l|} L_{p}^{|l|}\left(\frac{2 r^{2}}{w^{2}(z)}\right) e^{-r^{2} / w^{2}(z)}, \\
\Theta_{k l p}(\mathbf{R})=\frac{k r^{2} z}{2\left(z^{2}+z_{R}^{2}\right)}+l \phi+(2 p+l+1) \tan ^{-1}\left(z / z_{R}\right)+k z,
\end{gathered}
$$

Here $F_{k 00}$ may be identified as the amplitude for a plane wave propagating along the $z$-axis, with a wave-vector of magnitude $k$; the coefficient $N_{l p}$ is a normalization constant, and $w(z)$ is a characteristic width of the beam at axial coordinate $z$, explicitly given by $w^{2}(z)=2\left(z^{2}+z_{R}^{2}\right) / k z_{R}$, where $z_{R}$ is the Rayleigh range. The LG mode indices $l$ and $p$ determine the field intensity distribution and are such that $l \hbar$ is the orbital angular momentum conveyed by each photon. It is convenient at this stage to also introduce the position-dependent Rabi frequency $\Omega(\mathrm{R})$, defined in terms of the transition dipole moment $\mu_{12}$ as follows:

$$
\hbar \Omega(\mathbf{R})=\left|\left(\mu_{12} \cdot \hat{\varepsilon}\right) \alpha F(\mathbf{R})\right|,
$$

suppressing the indices klp. We also define

$$
f(\mathbf{R})=\Omega(\mathbf{R}) e^{\mathrm{i}(\mathbf{R})} .
$$

\section{B. Optical Bloch equations and their solution}

In order to set up the appropriate optical Bloch equations for the atomic density matrix elements we allow the position operator $\mathbf{R}$ and its canonically conjugate momentum operator $\mathbf{P}$ to be replaced by their average values, $\mathbf{r}$ and $\mathbf{P}_{0}=M \mathbf{V}$, where $\mathbf{V}$ is the center-of-mass velocity. This approximation amounts to treating the center-of-mass motion of the atom classically, while the internal dynamics continues to be treated quantum mechanically. This is acceptable provided the spread in the atomic wave packet is much smaller than the wavelength of the light, and that the recoil energy is much smaller than the linewidth. Within this approximation, the system density matrix associated with the two electronic levels and the center of mass can be written as $\rho s=\delta(\mathbf{R}-\mathbf{r}) \delta(\mathbf{P}-M \mathbf{V}) \rho(t)$ and the internal density matrix $\rho(t)$, accommodating dissipation through relaxation effects, satisfies the usual form:

$$
\frac{d \rho}{d t}=-\frac{\mathrm{i}}{\hbar}[H, \rho]+R \rho
$$

The optical Bloch equations for the two-level density matrix elements emerge from the above formalism in the following matrix form:

$$
\left[\begin{array}{c}
\dot{\hat{\rho}}_{21}(t) \\
\dot{\hat{\rho}}_{12}(t) \\
\dot{\rho}_{22}(t)
\end{array}\right]=\left[\begin{array}{ccc}
-\left(\Gamma_{2}-\mathrm{i} \Delta\right) & 0 & 2 f^{*}(r) \\
0 & -\left(\Gamma_{2}+\mathrm{i} \Delta\right) & 2 f^{*}(r) \\
-f^{*}(r) & -f(r) & -\Gamma_{1}
\end{array}\right]\left[\begin{array}{c}
\hat{\rho}_{21}(t) \\
\hat{\rho}_{12}(t) \\
\rho_{22}(t)
\end{array}\right]+\left[\begin{array}{c}
-f(r) \\
-f^{*}(r) \\
0
\end{array}\right]
$$


where the spontaneous emission process has been described in terms of an inelastic collision rate $\Gamma_{1}$, and an elastic collision rate $\Gamma_{2}$. Elastic collisions often have dominant line-broadening effects for a wide variety of physical conditions. We have also introduced $\Delta(\mathbf{R}, \mathbf{V})=\Delta_{0}-\mathbf{V} \nabla \Theta(\mathbf{R}, \mathbf{V})$, which is the effective, velocity-dependent detuning, and we set $\hat{\rho}=\tilde{\rho} \exp (-i t \mathbf{V} \cdot \nabla \boldsymbol{\Theta})$.

We use Laplace transform techniques to obtain time-dependent solutions to the optical Bloch equations, using a technique first employed by Torrey to solve nuclear Bloch equations. As shown elsewhere, ${ }^{13}$ the transformed density matrix elements take the form:

$$
\bar{P}(\lambda)=\frac{g(\lambda)}{\lambda|\Lambda|},
$$

where $\bar{P}$ represents either $\bar{\rho}_{12}, \bar{\rho}_{21}$, or $\bar{\rho}_{22}$, and $\lambda$ is the Laplace-transform (frequency) variable. Assuming that $|\Lambda|(\lambda)$ can be factorized in the form $|\Lambda|(\lambda)=(\lambda+a)\left[(\lambda+b)^{2}+s^{2}\right]$, then the results for $\bar{P}$ can be expressed as partial fractions:

$$
\bar{P}(\lambda)=\frac{A}{\lambda+a}+\frac{B(\lambda+b)+C}{(\lambda+b)^{2}+s^{2}}+\frac{D}{\lambda}
$$

Inverting the transform of this equation leads to solutions to optical Bloch equations that take the form:

$$
P(t)=A e^{-a t}+\left(B \cos (s t)+C s^{-1} \sin (s t)\right) e^{-b t}+D .
$$

Such solutions are known as Torrey solutions, in which the first three terms represent transient effects, and the final term the steady state. The aim is to seek time-dependent solutions in order to find the transient force exerted on a twolevel atom by a Laguerre-Gaussian beam. For this we need only $\bar{\rho}_{21}$, the only time-dependent factor entering the force expression, $\langle F(t)\rangle=\mathrm{i} \hbar \nabla\left[\tilde{\rho}_{21}^{*} f(\mathbf{r})-\tilde{\rho}_{21} f^{*}(\mathbf{r})\right]$. The corresponding results for $A, B, C$ and $D$ in (10) are as follows:

$$
\begin{gathered}
A=\frac{-g(-a)}{a\left[(b-a)^{2}+s^{2}\right]}, \\
D=\frac{g(0)}{a\left(b^{2}+s^{2}\right)}, \\
B=-(A+D), \\
C=a A+b B-f(r) .
\end{gathered}
$$

Dynamically distinct results can be secured for each of the regimes identified above, as determined by the extent of detuning, the relaxation parameters and the optical field strength. There are three special cases where the solution takes a simple form; those of $(a)$ exact resonance, $(b)$ strong collisions, and $(c)$ an intense external field.

(a) Exact resonance

$$
A=0 ; \quad B=\frac{f(\mathbf{r}) \Gamma_{1}}{\Gamma_{1} \Gamma_{2}+4|f(\mathbf{r})|^{2}} ; \quad C=\frac{f(\mathbf{r})\left(\Gamma_{1}^{2}-\Gamma_{1} \Gamma_{2}-8|f(\mathbf{r})|^{2}\right)}{2\left(\Gamma_{1} \Gamma_{2}+4|f(\mathbf{r})|^{2}\right)} ; \quad D=\frac{-f(\mathbf{r}) \Gamma_{1}}{\Gamma_{1} \Gamma_{2}+4|f(\mathbf{r})|^{2}} .
$$




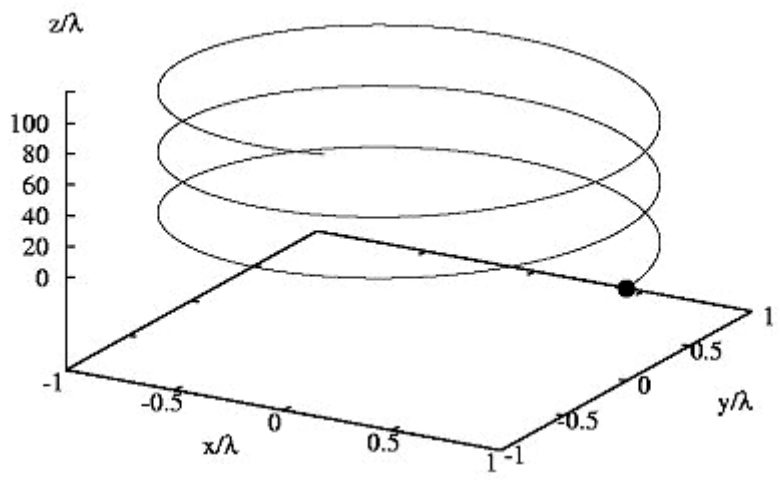

Fig. 1. Trajectory of an $\mathrm{Eu}^{3+}$ ion starting at rest in a $\mathrm{LG}_{1,0}$ mode, in the case of exact resonance. The dot represents the initial position of the atom. See the text for parameter values generating this trajectory. Adapted from ref. [13]

From the results for the ensuing dissipative force, it is expedient to develop illustrative trajectories. The result illustrated in Fig. 1, of helical form, is based on an $\mathrm{Eu}^{3+}$ ion (mass $25.17 \times 10^{-26} \mathrm{~kg}$ ) for which a suitable transition is ${ }^{5} D_{0} \rightarrow{ }^{7} D_{1}$, corresponding to a wavelength of $614 \mathrm{~nm}$. The upper state for this transition has $\Gamma=1111.11 \mathrm{~Hz}$ (we assume $\left.\Gamma=\Gamma_{2}=\Gamma_{1} / 2\right)$. We concentrate on the $l=1, p=0$ Laguerre-Gaussian mode, assuming an irradiance of $10^{5} \mathrm{~W} \mathrm{~cm}^{-2}$, and a beam waist $w_{0}=35 \lambda$. All the numerical evaluations whose results are presented here were run for a period $t \max \approx 5 \Gamma^{-1}$, which is approximately $4.5 \mathrm{~ms}$, a time sufficient for the transient regime to manifest itself in the results and also for the steady-state forces to be applicable by the end of the run.

(b) Strong collisions

$$
A=0 ; \quad B=\frac{f(\mathbf{r})(\Gamma+i \Delta)}{\Gamma^{2}+\Delta^{2}+4|f(\mathbf{r})|^{2}} ; \quad C=\frac{f(\mathbf{r})\left(i \Delta \Gamma-\Delta^{2}-4|f(\mathbf{r})|^{2}\right)}{\left(\Gamma^{2}+\Delta^{2}+4|f(\mathbf{r})|^{2}\right)} ; \quad D=\frac{-f(\mathbf{r})(\Gamma+i \Delta)}{\Gamma^{2}+\Delta^{2}+4|f(\mathbf{r})|^{2}}
$$

Here, the assumption $\Gamma=\Gamma_{2}=\Gamma_{1}$ secures the above results (16), and the corresponding ion trajectory is shown in Fig. 2.

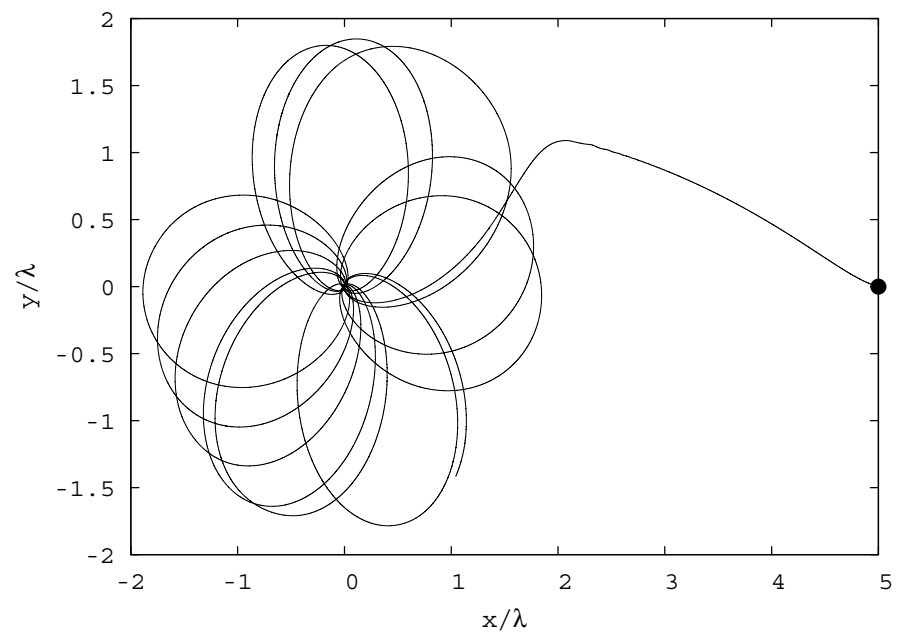

Fig. 2. Re-entrant trajectory of an $\mathrm{Eu}^{3+}$ ion projected in the $x-y$ plane, with the ion starting at rest in a $\mathrm{LG} 1,0$ mode, in the case of strong collisions. The dot represents the initial position of the ion. Adapted from ref. [13] 
(c) Intense external field

$$
A=\frac{f(\mathbf{r})\left(\Gamma_{2}+i \Delta-a\right)\left(\Gamma_{1}-a\right)}{a\left[(b-a)^{2}+s^{2}\right]} ; \quad B=-(A+D) ; \quad C=a A+b B-f(\mathbf{r}) ; \quad D=-\frac{f(\mathbf{r}) \Gamma_{1}\left(\Gamma_{2}+i \Delta\right)}{a\left(b^{2}+s^{2}\right)},
$$

with

$$
a=\Gamma_{2}-\left(\Gamma_{2}-\Gamma_{1}\right) \frac{\Delta^{2}}{\Delta^{2}+4|f(\mathbf{r})|^{2}} ; \quad b=\Gamma_{2}-\frac{2\left(\Gamma_{2}-\Gamma_{1}\right)|f(\mathbf{r})|^{2}}{\Delta^{2}+4|f(\mathbf{r})|^{2}} ; \quad s=\sqrt{\Delta^{2}+4|f(\mathbf{r})|^{2}}
$$

For an irradiance of $10^{5} \mathrm{~W} \mathrm{~cm}{ }^{-2}$, and with $\Gamma=\Gamma_{2}=\Gamma_{1} / 2$, the ion trajectory is as shown in Fig. 3. It is observed both in the cases of strong collisions and of an intense external field that the atomic trajectory follows a characteristic path, in axial motion is superimposed on an in-plane motion. The re-entrant, in-plane trajectory is in the form of loops resembling the petals of a flower.

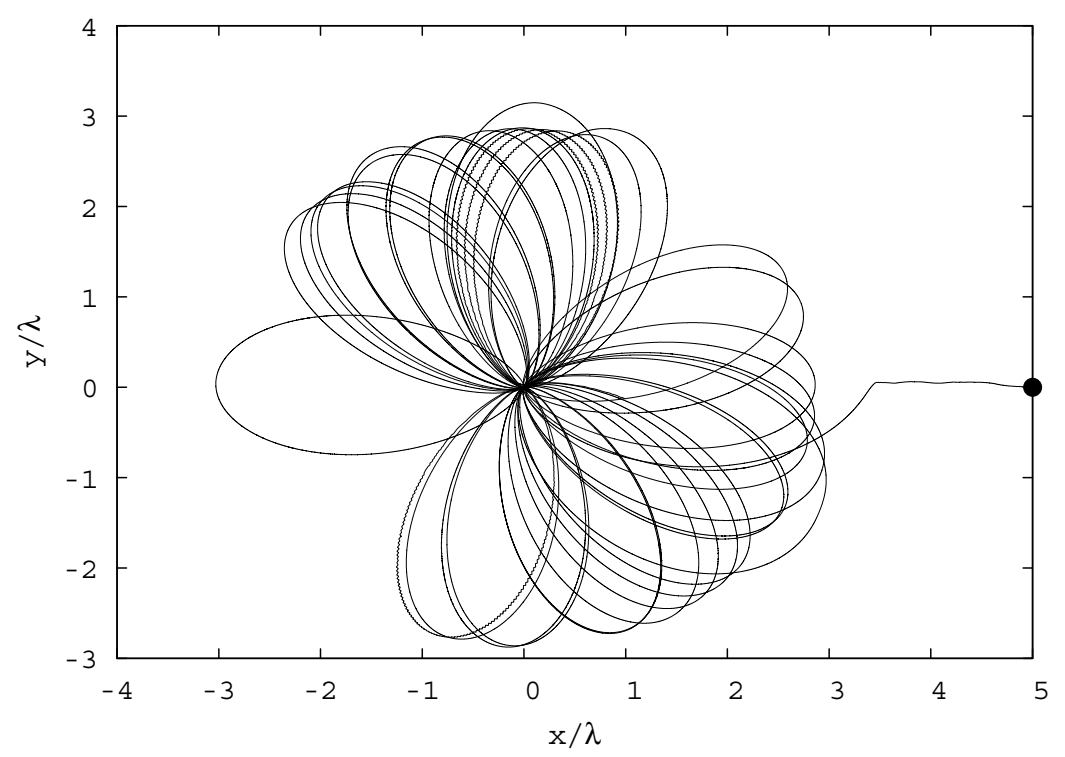

Fig. 3. Trajectory of an $\mathrm{Eu}^{3+}$ as in Fig. 2, but here for the case of a strong external field. Adapted from ref. [13]

\section{ATOM TRAPPING IN MULTIPLE TWISTED BEAMS}

\section{A. Beam representation}

In this section, the aim is to consider configurations of multiple twisted beams propagating in orientationally arbitrary, but specified, directions. A twisted beam propagating in a general direction can be constructed from the standard one, described in Section 2A, by the following transformations between rotated Cartesian axes, with coordinates on a common origin recast in cylindrical polar form:

$$
\left.\begin{array}{l}
x \rightarrow x^{\prime}=\cos (\theta) x+\sin (\theta) z \\
y \rightarrow y^{\prime}=-\sin (\theta) \sin (\psi) x+\cos (\psi) y+\cos (\theta) \sin (\psi) z \\
z \rightarrow z^{\prime}=-\sin (\theta) \cos (\psi) x+\sin (\psi) y+\cos (\theta) \cos (\psi) z
\end{array}\right\}
$$

The equation of motion for an atom or ion at the intersection of two or more beams is then determined using; 


$$
M \frac{\partial^{2}}{\partial t^{2}} \mathbf{R}(t)=\sum_{i}\left\langle\mathbf{F}_{i}\right\rangle+q \mathbf{V} \times \mathbf{B}
$$

where $q$ is the charge, $\mathrm{V}=\partial \mathrm{R} / \partial t$ is the velocity, and $\mathrm{B}$ is the magnetic field, if present.

\section{B. Two-dimensional optical molasses}

We consider first a beam geometry corresponding to a generalised two-dimensional optical molasses configuration, in which four counter-propagating beams effect a planar trap, ${ }^{14}$ but where all four beams are twisted. The forces acting on the atom are a sum of contributions from each of the beams, where the correct specifications of three of them must be made using the transformation equations (19). Calculations are performed for two $\mathrm{Mg}^{+}$ions initially positioned at different points, with each having an initial velocity of $v_{z}=5 \mathrm{~m} \mathrm{~s}^{-1}$, for the case where each of the four beams has an azimuthal index, $l=1$, and radial index $p=0$. The ion transition frequency corresponds to a wavelength of $280.1 \mathrm{~nm}$ and the transition rate is $2.7 \times 10^{8} \mathrm{~s}^{-1}$; the mass of $\mathrm{Mg}^{+}$is $4.0 \times 10^{-26} \mathrm{~kg}$. Since, by choice of $l$ values in this case, the torques due to both pairs of beams are zero, each ion ends up at a specific fixed point, where it remains essentially motionless on the locus of lowest minima (corresponding to the two oblique orthogonal circles shown in Fig. 4).

When a third pair of counter-propagating beams is added to the two-dimensional configuration, orthogonal to the plane containing the original beams, we have a three-dimensional configuration. In this case the deepest potential minima are located at eight discrete points defined by the coordinates: $x= \pm 1 / 2 w_{0}, y= \pm 1 / 2 w_{0}, z= \pm 1 / 2 w_{0}$, signifying the eight corners of a cube of side $w_{0}$, centred on the origin, as shown in Fig. 5.

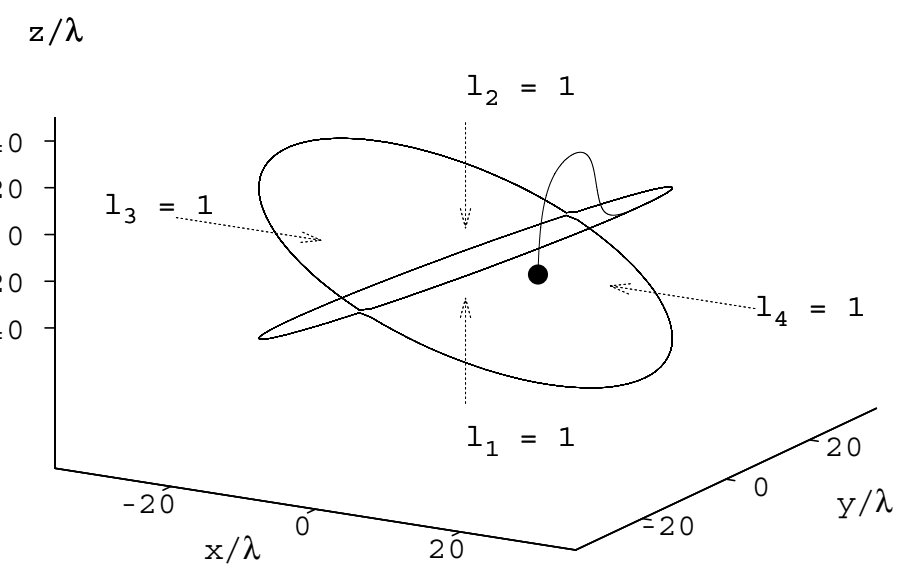

Fig. 4. Trajectories of two $\mathrm{Mg}^{+}$ions starting from different positions in counter-propagating twisted beams, $i=1-4\left(l_{i}=1, p_{i}=0\right)$.

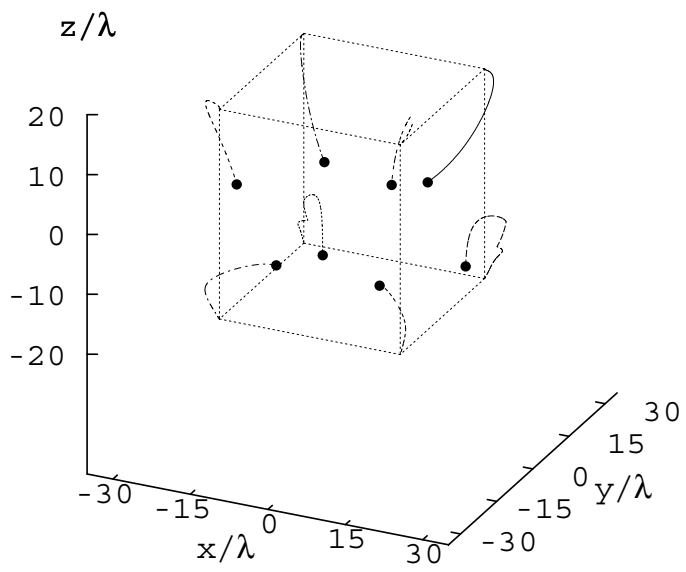

Fig. 5. The trajectories of $\mathrm{Mg}^{+}$ions in 3D counter-propagating LG beams, $i=1-6\left(l_{i}=1, p_{i}=0\right)$. 


\section{DISCUSSION}

Our calculations of atomic trajectories in a twisted optical beam show features that vary dramatically according to the extent of frequency detuning, the relaxation conditions and the optical field strength. In the cases of strong collisions, and of an intense external field, the atomic trajectory typically follows a path in which there is axial motion superimposed on an in-plane motion. However, in the intense field case the number of loops is greatly increased; this is due to the increase in kinetic energy of the atom, both translational (radially due to stronger radial forces) as well as rotationally (due to the torque) in the more intense Laguerre-Gaussian beam. The looped trajectory cannot arise in the case of exact resonance, since there is no dipole force acting on the atom, and hence no radial force, due to the zero detuning. In this special case the radial position remains constant, as is exhibited in the results of model calculations shown in Fig. 1. Clearly, transient effects due to the interaction of atoms with a Laguerre-Gaussian beam become significant when the upper-state lifetime is relatively long. The evolution of the dynamical variables of the gross motion are markedly different from those that would be predicted from the steady-state forces. The trajectories determined here suggest that, under certain circumstances, consideration of the transient regime is crucial for correct representation of the dynamics. Transient behavior should certainly be taken into account in the optical manipulation of atomic beams, especially when tuning close to transitions with a long excited state lifetime.

Suppressing $z$-motion with a counter-propagating beam optical molasses configuration, atomic trapping becomes possible. Unlike Gaussian light, twisted light has a radial force which can be employed to keep an atom trapped within the field of the beams. The results highlight the potential significance of using twisted light in cooling and trapping of atoms and ions. Moreover the method used, which ensures proper speciation of the important characteristics of twisted beams, is applicable to a variety of configurations by appropriately defining the orientation of each beam with respect to the rest. Beyond the cubic configuration achievable with six counter-propagating beams, depicted in Fig. 5, one can envisage the creation of larger optical lattices, allowing periodic replication of the twisted molasses arrangement. We suggest that the formation of a finite, non-interacting atomic lattice might usefully be exploited in the study of coupled Bose-Einstein condensates (BECs) and, as in the case of plane wave optical lattices, for fundamental quantum information studies. ${ }^{15}$ Evaporative cooling techniques could then be followed to produce the BECs. ${ }^{16}$ Progressively stronger coupling between trapped BECs would be achievable by a reduction in the value of $w_{0}$. The twisted beam environment offers flexibility, in that one has a considerable freedom of choice over the various parameters, for example controlling the distances between the trapping sites by varying the beam waist, while the depth of the optical potential is determined by the light intensity and the detuning. The most obvious advantage over conventional ion traps is its alloptical implementation, and its amenability for use for either electrically charged or neutral particles.

\section{ACKNOWLEDGMENT}

The authors gratefully acknowledge that this work is funded by the U.K. Engineering and Physical Sciences Research Council.

\section{REFERENCES}

1. C. S. Adams and E. Riis, Prog. Quantum Electron. 21, 1 (1997).

2. V. S. Letokhov and V. G. Minogin, Laser Light Pressure on Atoms, Gordon and Breach, New York, 1987.

3. A. P. Kazantsev, G. I. Surdutovich, and V. P. Yakovlev, Mechanical Action of Light on Atoms, World Scientific, Singapore, 1990.

4. M. Babiker, W. L. Power, and L. Allen, Phys. Rev. Lett. 73, 1239 (1994).

5. L. Allen, M. Babiker, W. K. Lai, and V. E. Lembessis, Phys. Rev. A 54, 4259 (1996).

6. M. E. J. Friese, T. A. Nieminen, N. R. Heckenberg, and H. Rubinsztein-Dunlop, Nature (London) 394, 348 (1998).

7. H. He, M. E. J. Friese, N. R. Heckenberg, and H. Rubinsztein-Dunlop, Phys. Rev. Lett. 75, 826 (1995).

8. M. Babiker, C. R. Bennett, D. L. Andrews, and L. C. Davila Romero, Phys. Rev. Lett. 89, 143601 (2002).

9. J. Courtial, K. Dholakia, L. Allen, and M. J. Padgett, Opt. Commun. 144, 210 (1997).

10. A. T. O’Neil, I. MacVicar, L. Allen, and M. J. Padgett, Phys. Rev. Lett. 88, 053601 (2002).

11. L. Allen, M. J. Padgett, and M. Babiker, Prog. Opt. 34, 291 (1999). 
12. L. Allen, S. M. Barnett, and M. J. Padgett, Optical Angular Momentum, Institute of Physics Publishing, Bristol, 2003.

13. A. R. Carter, M. Babiker, M. Al-Amri and D. L. Andrews, Phys. Rev. A 72, 043407 (2005).

14. V. I. Balykin, V. G. Minogin and V. S. Letokhov, Rep. Prog. Phys. 63, 1429 (2000).

15. D. Bouwmeester, A. Ekert, and A. Zeilinger, eds., The Physics of Quantum Information, Springer, Berlin, 2000.

16. C. J. Pethick and H. Smith, Bose-Einstein Condensation in Dilute Gases, Cambridge University Press, Cambridge, 2002.

*david.andrews@physics.org 